Introduction Aberration of MLN64 (Metastatic Lymph Node Protein 64), also known as StARD3 (StAR Related Lipid Transfer Domain Containing 3), has been reported in endocrine related cancers including breast and prostate cancers and found to be present in insulinoma cells. The present study evaluated the clinical and prognostic values of MLN64 to clarify its role in human pancreatic cancer.

Methods Human pancreatic cancer cell lines, PANC1 and MIA PaCa-2 were used. The levels of MLN64 gene transcripts were quantitatively evaluated in a cohort of human pancreatic cancer with matched normal tissues. The expression of the transcripts were evaluated against the clinical, pathological and outcome parameters of the patients.

Results Pancreatic cancer tissues had a markedly increased levels of MLN64 (Median (Q1-Q3)): 4.85 (0-61) compared with normal tissues $(0.03(0-7.6))(\mathrm{p}<0.001)$. It was also revealed that MLN64 in tumours of patients who died of pancreatic cancer during the followup period had a significantly highly levels of MLN64 (7.66 (0-20)), compared with those who survived $(0.02(0-72.5))(\mathrm{p}<0.01)$, further indicating MLN64 as a significant prognostic factor (HR 8.37, $\mathrm{p}<0.01)$. Our data also revealed node positive pancreatic tumours had a significantly higher levels of MLN64 than node negative tumours $(p<0.05)$, although no significant difference was seen between those with distant metastases and those without. Significant correlation was also found between MLN64 and the Her family members, namely Her4 $(\mathrm{p}<0.001)$ and Her3 $(\mathrm{p}<0.05)$ and that the combining the pattern of expression of MLN64 and Her4 further enhance the power in predicting outcomes of the patients. Using our pancreatic cancer cell models, we have demonstrated that blocking Her family kinases using a panHer inhibitor, namely Nerlynx and a key cell migration regulator, phospholipase C-gamma1 (PLC $\square$ 1), had a marked impact on cellular migration and adhesion of pancreatic cancer cells, key functions observed with MLN64 in cancer cells.

Conclusions MLN64/StARD3, one of the key regulators of cancer cells, has an aberrant expression in human pancreatic cancer which is linked to the clinical outcome. With its role in cancer cells and discovery of inhibitory means to MLN64/ StARD3, the molecule presents a good target for therapeutic considerations in pancreatic cancer.

\section{PTU-69 AUGMENTING THE EFFECT OF SONODYNAMIC THERAPY AGAINST PANCREATIC CANCER USING HEMATOPORPHYRIN-CARRYING NANOPARTICLES}

${ }^{1}$ Theo Reback* ${ }^{1}$ Maryam Hadi, ${ }^{1}$ Alexander Ng, ${ }^{1}$ Bala Ramesh, ${ }^{2} J o h n$ Callan, ${ }^{1}$ Alexander MacRobert, ${ }^{2}$ Anthony McHale, Nikolitsa Nomikou. ${ }^{1}$ Division of Surgery and Interventional Science, Faculty of Medical Sciences, London, UK; ${ }^{2}$ Biomedical Sciences Research Institute, Coleraine, UK

\subsection{6/gutjnl-2021-BSG.271}

Introduction Sonodynamic therapy (SDT) is an emerging, minimally invasive therapeutic approach with the potential to treat a variety of recalcitrant cancers, including pancreatic cancer. The treatment involves the use low intensity ultrasound in combination with non-toxic agents, known as sonosensitizers, for the site-specific ablation of tumours. After systemic administration of the sensitizing agent, the application of ultrasound can be performed extracorporeally, endoscopically or intraoperatively. This study evaluates the effectiveness of SDT using a new nanoparticulate formulation carrying hematoporphyrin in the in vitro treatment of human pancreatic cancer cells.
Methods The human BxPC-3 cell line was used as an in vitro model for pancreatic adenocarcinoma. The target cells were incubated with free hematoporphyrin (free HP) and hematoporphyrin-containing nanoparticles (HPNP) that were formed by self-assembly with a polyglutamate-tyrosine co-polymer, in order to determine the cytotoxicity in the absence of ultrasound. Cells were treated with HPNP combined with ultrasound irradiation for determining the effect of SDT. These effects were examined at normoxic and hypoxic conditions, at $\mathrm{pH} 6.4$ and $\mathrm{pH}$ 7.4.

Results The HPNP nanoparticles showed increased toxicity against the target cell line, when compared with free HP. The HPNP toxicity was further enhanced at acidic conditions and this is particularly important for confining the ablation effect within the acidic pancreatic tumour mass. Utilising the nanoparticle carrier, cellular uptake of hematoporphyrin was significantly increased compared to the free HP $(\mathrm{p}<0.0001)$, at both acidic and physiological $\mathrm{pH}$. SDT, at varying ultrasound treatment conditions and at both normoxic and hypoxic environment, demonstrated a clear cytotoxic effect against the BxPC-3 cell line $(<0.001)$, while toxicity of ultrasound alone or the nanoparticles alone was minimal.

Conclusion BxPC-3 cells have significant positive response to treatment with SDT. Further preclinical experimentation is currently being carried out in experimental animals to evaluate the effect of SDT in vivo for supporting the clinical translation of this promising therapeutic modality in the treatment of pancreatic cancer.

\section{PTU-70 PANCREATIC ENZYME REPLACEMENT IN UNRESECTABLE PANCREATIC CANCER: A RETROSPECTIVE STUDY FROM A DISTRICT GENERAL HOSPITAL}

Timothy Keen*, Melissa Zhao, Keith Chan, Lachlan Ayres. University Hospital Dorset, Poole, UK

\subsection{6/gutjnl-2021-BSG.272}

Introduction Advanced pancreatic cancer has a poor prognosis and is associated with pancreatic exocrine insufficiency (PEI) causing weight loss and cachexia. Pancreatic enzyme replacement therapy (PERT) is recommended by the National Institute for Health and Care Excellence (NICE) for patients with unresectable pancreatic cancer but is often not prescribed despite evidence it can improve quality of life and survival. The aim of this retrospective study was to assess adherence to the NICE recommendations on the use of PERT and its impact on patient survival.

Methods Patients with a radiological or histological diagnosis of unresectable pancreatic cancer were identified from a retrospective cancer multidisciplinary team database in a district general hospital from January 2017 to August 2019. Patient demographics and survival from time of diagnosis were compared for patients treated with and without PERT. T-tests and Chi-squared tests were used as appropriate.

Results 166 patients diagnosed with unresectable pancreatic cancer were identified, 80 (48\%) were treated with PERT. Median age was 75 years in the PERT group versus 79 years in the no PERT group $(\mathrm{p}=.014)$.

The PERT group were more likely to have better performance status (performance status $\geq 3,33 \%$ vs $56 \% \quad(p=0.024)$, less likely to have advanced disease (stage $\geq 3,33 \%$ vs $56 \%$ 


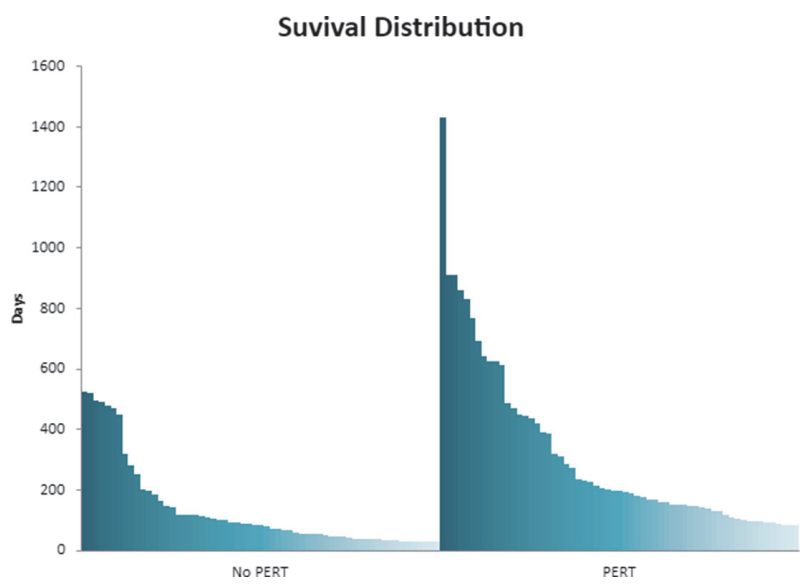

Abstract PTU-70 Figure 1

$(p=.0017)$ and were more likely to receive chemotherapy (33 patients vs 12 patients, $\mathrm{p}<.001)$ compared to the no PERT group. The PERT group had longer survival times (median survival 151 days vs 46 days, $\mathrm{p}<.001$, figure 1 ).

There was increased survival for patients treated with PERT when patients treated with chemotherapy were excluded (median survival of 98 days vs 37 days, $\mathrm{p}=.004$ ). Additionally, there was increased survival for those treated with PERT and chemotherapy compared to those treated with chemotherapy without PERT (226 days vs 101 days, $\mathrm{p}=.04$ ).

Sub-group analysis demonstrated statistically significant increased survival for patients treated with PERT with cancer of the pancreatic head (median survival of 165 days vs 44 days, $\mathrm{p}=.001$ ) and of the pancreatic body (median survival of 174 days vs 37 days, $\mathrm{p}=.001)$.

Conclusions This retrospective, pragmatic study of patients diagnosed with unresectable pancreatic cancer showed that less than half of patients were treated with PERT. PERT was associated with a clinically significant (approximately 3-fold) increase in survival regardless of whether chemotherapy was given. However, these results are likely to be confounded by selection bias given the higher percentage of patients in the no PERT group with more advanced disease and poorer performance status. We recommend offering all patients diagnosed with unresectable pancreatic cancer PERT.

\section{PTU-71 POST ERCP INDUCED PANCREATITIS: DOES PROPHYLACTIC PANCREATIC STENTING MAKE A DIFFERENCE?}

Brenavan Natarajan*, Bijan Yazdanian, Akash Jha, Alistair King, Mohammed Shariff, Anthony Leahy. Watford General Hospital, Watford, UK

\subsection{6/gutjnl-2021-BSG.273}

Introduction Post- ERCP Pancreatitis (PEP) is a significant complication of ERCP, especially in high risk rases or where there has been involvement of the pancreatic duct. Rectal NSAIDs and pancreatic duct stents are used to reduce the risk of PEP. It is not yet known if PD stents plus rectal NSAIDs reduce the risk of PEP more than rectal NSAIDs alone.

Methods We performed a retrospective analysis of 2028 ERCPs performed at a single large DGH centre in the UK to examine if PD stenting plus rectal NSAID significantly reduced the risk post procedure complications.

\begin{tabular}{|c|c|c|c|}
\hline & $\begin{array}{l}\text { No PD wire } \\
\text { or contrast }\end{array}$ & $\begin{array}{l}\text { PD wire or contrast } \\
\text { but no PD stent }\end{array}$ & $\begin{array}{l}\text { PD wire or contrast } \\
\text { and PD stent inserted }\end{array}$ \\
\hline Total number & 1469 & 348 & 211 \\
\hline Mean age in years & 69 & 67 & 64 \\
\hline Range in years & $16-101$ & $17-101$ & $19-94$ \\
\hline Male: Female ratio & $628: 841$ & 151:197 & $87: 124$ \\
\hline ASA Status & 1.48 & 1.40 & 1.80 \\
\hline Pancreatitis rate & $\begin{array}{l}36 / 1469 \\
(2.5 \%) \\
(p=0.01)\end{array}$ & $\begin{array}{l}15 / 348 \\
(4.3 \%) \\
(p=0.03)\end{array}$ & $\begin{array}{l}10 / 211 \\
(4.7 \%) \\
(p=0.03)\end{array}$ \\
\hline $\begin{array}{l}\text { Mean number of PD } \\
\text { contrast injections }\end{array}$ & $\mathrm{n} / \mathrm{a}$ & 0.55 & 0.64 \\
\hline $\begin{array}{l}\text { Mean number of PD } \\
\text { wire passes }\end{array}$ & $\mathrm{n} / \mathrm{a}$ & 1.87 & 2.85 \\
\hline $\begin{array}{l}\text { IV fluids administered } \\
\text { post procedure }\end{array}$ & $11 \%$ & $0 \%$ & $0 \%$ \\
\hline Rectal NSAID given & $36 / 36(100 \%)$ & $15 / 15(100 \%)$ & $10 / 10(100 \%)$ \\
\hline $\begin{array}{l}\text { Severity of } \\
\text { pancreatitis } \\
\text { (Modified Marshall } \\
\text { score) }\end{array}$ & 0.97 & $0.4(p=0.13)$ & $0.6(p=0.53)$ \\
\hline $\begin{array}{l}\text { Mean Length of stay } \\
\text { (days) }\end{array}$ & 7.7 & $6.9(p=0.70)$ & $5.5(p=0.11)$ \\
\hline ITU involvement & $4 / 36(11 \%)$ & $2 / 15(13 \%)$ & $0 / 10(0 \%)$ \\
\hline Deaths & $4 / 36(11 \%)$ & $1 / 15(6.7 \%)$ & $0 / 10(0 \%)$ \\
\hline
\end{tabular}

Results We found pancreatitis occurred in 3\% of ERCPs $(n=61)$. All patients had rectal NSAIDs administered at time of ERCP. We found PD involvement significantly increased the rate of PEP. We found that PD stenting did not significantly provide reduction in the severity of pancreatitis, measured by Modified Marshal or Atlanta scores, compared to those that had PD involvement without PD stent insertion or those without PD duct involvement. There was no significant difference in length of admission amongst groups.

Conclusions Our study further demonstrates that PD involvement at ERCP increases the rate of PEP. However, our study suggests PD stenting does not significantly provide reduction in rates of pancreatitis when used in combination with rectal NSAID therapy. In addition, there is an additional cost associated with PD stent placement plus the need for further follow-up if the PD stent does not spontaneously pass. We do reflect the number of pancreatitis cases in our cohort was small and that this was the experience from a single, large centre. We suggest further randomised controlled trials are required to prove the benefit of $\mathrm{PD}$ stent placement in combination with rectal NSAIDs over rectal NSAIDs alone in prevention of post-ERCP pancreatitis.

\section{PTU-72 OUTCOMES OF SURGERY FOR CHRONIC PANCREATITIS IN THE MANCHESTER SPECIALIST MULTI-DISCIPLINARY PANCREATITIS SERVICE}

Matthew Burrows*, Ala Elwasila, Ajith Siriwardena, Alistair Makin, Abubaker YM Ahmed. Manchester University Foundation Trust, Manchester, UK

\subsection{6/gutjnl-2021-BSG.274}

Introduction The management of chronic pancreatitis is complex. In Manchester, a multi-disciplinary approach to care has 